\title{
Obtaining an Initial Solution for Facility Layout Problem
}

\author{
Ali Shoja Sangchooli and Mohammad Reza Akbari Jokar \\ Department of Industrial Engineering, Sharif University of Technology, Tehran 1458875346, Iran \\ Correspondence should be addressed to Ali Shoja Sangchooli; a_shojasangchooli@ie.sharif.edu \\ Received 13 April 2013; Accepted 29 August 2013 \\ Academic Editor: Ting Chen
}

Copyright (c) 2013 A. Shoja Sangchooli and M. R. Akbari Jokar. This is an open access article distributed under the Creative Commons Attribution License, which permits unrestricted use, distribution, and reproduction in any medium, provided the original work is properly cited.

\begin{abstract}
The facility layout approaches can generally be classified into two groups, constructive approaches and improvement approaches. All improvement procedures require an initial solution which has a significant impact on final solution. In this paper, we introduce a new technique for accruing an initial placement of facilities on extended plane. It is obtained by graph theoretic facility layout approaches and graph drawing algorithms. To evaluate the performance, this initial solution is applied to rectangular facility layout problem. The solution is improved using an analytical method. The approach is then tested on five instances from the literature. Test problems include three large size problems of 50, 100, and 125 facilities. The results demonstrate effectiveness of the technique especially for large size problems.
\end{abstract}

\section{Introduction}

The facility layout problem seeks the best positions of facilities to optimize some objective. The common objective is to reduce material handling costs between the facilities. The problem has been modeled by a variety of approaches. A detailed review of the different problem formulations can be found in Singh and Sharma [1]. The facility layout problem is an optimization problem which arises in a variety of problems such as placing machines on a factory floor, VLSI design, and layout design of hospitals, schools.

The facility layout approaches can generally be classified into two groups, constructive methods and improvement methods. In this paper, we consider the placement of facilities on an extended plane. Many improvement approaches have been proposed for this problem. All improvement procedures require an initial solution. Some approaches start from a good but infeasible solution [2-4]. These models contain a penalty component in their objective function. Hence, these approaches minimize objective function value for feasible solutions. But some approaches require a feasible initial solution. These approaches use a randomly generated initial solution [5, 6]. Mir and Imam [7] have proposed simulated annealing for a better initial solution. They have shown that a good initial solution has a significant impact on final solution.

In this paper, we introduce a new technique for accruing an initial placement of facilities on an extended plane. The technique consists of two stages. In the first stage, a maximal planar graph (MPG) is obtained. In the second stage, the vertices of MPG are drawn on the plane by graph drawing algorithms. Then, vertices are replaced by facilities. Hence, an initial solution is obtained.

In an MPG, the facilities with larger flows are adjacent together. Hence, drawing the MPG on the plane can be a good idea for obtaining an initial solution. To evaluate the performance of the idea, this initial solution is applied in rectangular facility layout problem. The solution is improved by an analytical method by Mir and Imam [7]. The approach is then tested on five instances from the literature.

The remaining parts of the paper are organized as follows. The next section describes the formulation of the facility layout problem chosen for our work. Section 3 describes accruing an initial placement. In Section 4 , the analytical method is described, and the approach is compared to other approaches in the literature. In Section 5, the proposed initial solution is compared with random initial solution. Finally, Section 6 provides a summary and conclusion. 


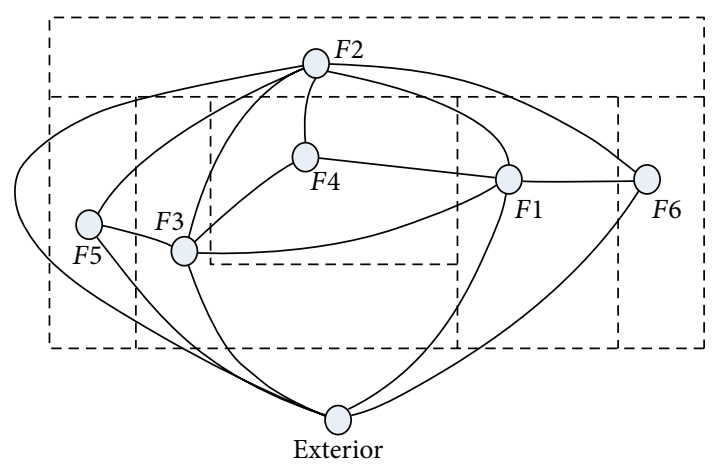

FIGURE 1: An MPG (solid lines) and its correspondent block layout (dashed lines).

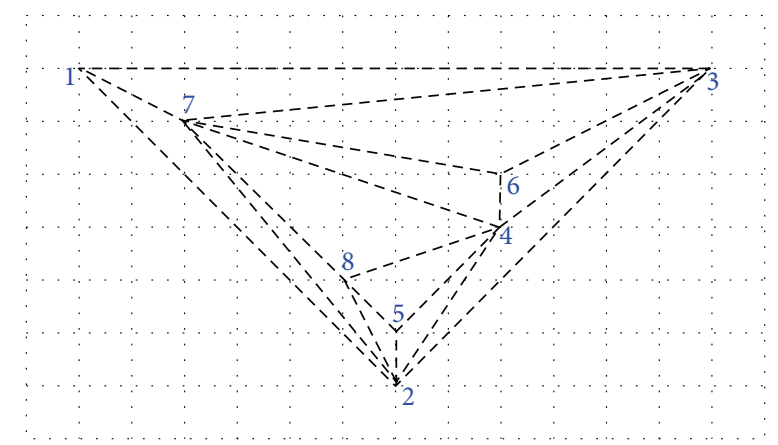

FIGURE 2: An example of straight line drawing.

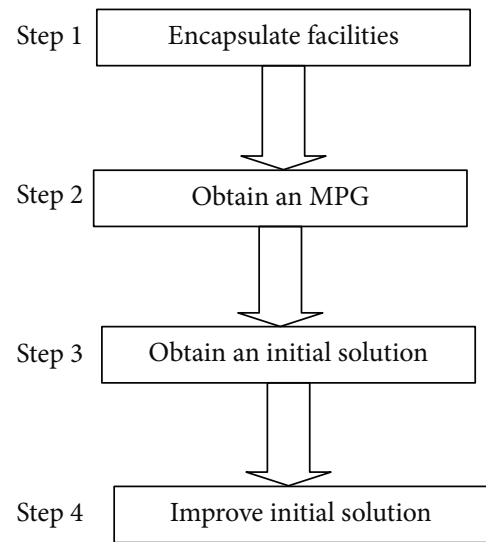

Figure 3: Steps of the proposed approach.

\section{Problem Formulation}

In this paper, we label the facilities $1,2, \ldots, N$, where $N$ is the total number of facilities. Facilities are assumed to be rectangles with fixed shape. The notation is given as follows:

$\left(x_{i}, y_{i}\right)$ coordinates of the center of facility $i$

$L_{i}$ length of facility $i$

$W_{i}$ width of facility $i$

$f_{i j}$ the total cost of flow per unit distance between two facilities $i$ and $j$
TABLE 1: Results for test problem \#1.

\begin{tabular}{lc}
\hline Program & Cost function value \\
\hline TOPOPT (Imam and Mir, 1989) [5] & 794 \\
VIP-PLANOPT (2006) [8] & 692 \\
GOT & 752.7 \\
\hline
\end{tabular}

TABLE 2: Results for test problem \#2.

\begin{tabular}{lc}
\hline Program & Best design \\
\hline Topopt (Mir and Imam, 1989) [5] & 1320.72 \\
FLOAT (Imam and Mir, 1993) [6] & 1264.94 \\
HOT (Imam and Mir, 2001) [7] & 1225.40 \\
VIP-PLANOPT (2006) [8] & 1157 \\
GOT & 1302 \\
\hline
\end{tabular}

TABLE 3: Results for test problem \#3.

\begin{tabular}{lc}
\hline Program & Cost function value \\
\hline HOT (Mir and Imam, 2001) [7] & 80794.24 \\
VIP-PLANOPT (2006) [8] & 78224.7 \\
GOT & 76882.3
\end{tabular}

TABLE 4: Results for test problem \#4.

\begin{tabular}{lc}
\hline Program & Cost function value \\
\hline HOT (Mir and Imam, 2001) [7] & 558556.2 \\
VIP-PLANOPT (2006) [8] & 538193.1 \\
GOT & 527094.1 \\
\hline
\end{tabular}

$d_{i j}$ distance between the centers of the facilities $i$ and $j$. $d_{i j}$ could be one of the following three distance norms.

(1) Euclidean distance:

$$
d_{i j}=\left(\left(x_{i}-x_{j}\right)^{2}+\left(y_{i}-y_{j}\right)^{2}\right)^{1 / 2} .
$$

(2) Squared Euclidean distance:

$$
d_{i j}=\left(x_{i}-x_{j}\right)^{2}+\left(y_{i}-y_{j}\right)^{2} .
$$

(3) Rectilinear distance:

$$
d_{i j}=\left|y_{i}-y_{j}\right|+\left|x_{i}-x_{j}\right| \text {. }
$$

The requirement for problem is that the facilities must not overlap each other. The area of overlap is defined as follows:

$$
A_{i j}=\lambda_{i j}\left(\Delta X_{i j}\right)\left(\Delta Y_{i j}\right) \text {, }
$$

where

$$
\begin{gathered}
\Delta X_{i j}=\frac{L_{i}+L_{j}}{2}-\left|x_{i}-x_{j}\right|, \\
\Delta Y_{i j}=\frac{W_{i}+W_{j}}{2}-\left|y_{i}-y_{j}\right|, \\
\lambda_{i j}= \begin{cases}1, & \text { for } \Delta X_{i j} \geq 0, \Delta Y_{i j} \geq 0, \\
0, & \text { otherwise. }\end{cases}
\end{gathered}
$$


TABLE 5: For test problem \#4, the coordinates of facilities obtained by GOT are given below. The value of the cost function for this layout is 527094.1 .

\begin{tabular}{|c|c|c|}
\hline Facility & $X$ & $Y$ \\
\hline 1 & 17.599 & 22.158 \\
\hline 2 & 18.514 & 32.933 \\
\hline 3 & 29.485 & 18.634 \\
\hline 4 & 35.526 & 20.582 \\
\hline 5 & 14.798 & 22.65 \\
\hline 6 & 26.425 & 25.388 \\
\hline 7 & 12.213 & 13.282 \\
\hline 8 & 19.435 & 27.466 \\
\hline 9 & 32.119 & 15.319 \\
\hline 10 & 24.743 & 8.862 \\
\hline 11 & 16.231 & 2.711 \\
\hline 12 & 25.533 & 17.621 \\
\hline 13 & 14.274 & 20.352 \\
\hline 14 & 22.791 & 3.889 \\
\hline 15 & 14.467 & 14.812 \\
\hline 16 & 38.012 & 16.94 \\
\hline 17 & 9.735 & 11.276 \\
\hline 18 & 24.609 & 27.663 \\
\hline 19 & 20.685 & 20.578 \\
\hline 20 & 19.866 & 22.579 \\
\hline 21 & 15.832 & 8.421 \\
\hline 22 & 8.866 & 31.495 \\
\hline 23 & 22.402 & 17.335 \\
\hline 24 & 35.551 & 9.125 \\
\hline 25 & 16.95 & 19.378 \\
\hline 26 & 36.104 & 25.31 \\
\hline 27 & 16.328 & 21.843 \\
\hline 28 & 13.208 & 26.161 \\
\hline 29 & 22.439 & 14.732 \\
\hline 30 & 14.052 & 13.065 \\
\hline 31 & 28.21 & 21.934 \\
\hline 32 & 16.436 & 12.282 \\
\hline 33 & 24.467 & 11.365 \\
\hline 34 & 23.103 & 32.489 \\
\hline 35 & 23.283 & 24.968 \\
\hline 36 & 19.444 & 14.708 \\
\hline 37 & 32.927 & 16.699 \\
\hline 38 & 11.708 & 28.857 \\
\hline 39 & 15.14 & 29.793 \\
\hline 40 & 28.164 & 5.174 \\
\hline 41 & 32.08 & 8.476 \\
\hline 42 & 29.636 & 13.049 \\
\hline 43 & 26.373 & 31.127 \\
\hline 44 & 8.693 & 15.785 \\
\hline 45 & 16.05 & 26.859 \\
\hline 46 & 31.965 & 26.099 \\
\hline 47 & 29.361 & 9.586 \\
\hline 48 & 33.822 & 12.885 \\
\hline
\end{tabular}

TABle 5: Continued.

\begin{tabular}{|c|c|c|}
\hline Facility & $X$ & $Y$ \\
\hline 49 & 19.394 & 25.043 \\
\hline 50 & 25.902 & 21.954 \\
\hline 51 & 4.234 & 16.534 \\
\hline 52 & 19.539 & 30.211 \\
\hline 53 & 5.587 & 11.801 \\
\hline 54 & 13.399 & 32.831 \\
\hline 55 & 28.771 & 16.071 \\
\hline 56 & 30.974 & 22.774 \\
\hline 57 & 22.453 & 22.117 \\
\hline 58 & 12.35 & 23.138 \\
\hline 59 & 21.725 & 18.944 \\
\hline 60 & 30.419 & 31.854 \\
\hline 61 & 24.388 & 18.897 \\
\hline 62 & 19.587 & 36.974 \\
\hline 63 & 19.943 & 12.839 \\
\hline 64 & 24.632 & 15.019 \\
\hline 65 & 5.951 & 32.233 \\
\hline 66 & 16.918 & 14.346 \\
\hline 67 & 23.818 & 6.408 \\
\hline 68 & 19.581 & 18.447 \\
\hline 69 & 35.191 & 31.263 \\
\hline 70 & 0.927 & 21.347 \\
\hline 71 & 32.644 & 19.464 \\
\hline 72 & 9.487 & 20.325 \\
\hline 73 & 39.749 & 21.788 \\
\hline 74 & 18.485 & 16.27 \\
\hline 75 & 15.581 & 36.344 \\
\hline 76 & 6.345 & 20.203 \\
\hline 77 & 13.484 & 23.21 \\
\hline 78 & 18.527 & 20.734 \\
\hline 79 & 11.773 & 20.295 \\
\hline 80 & 19.329 & 2.663 \\
\hline 81 & 4.471 & 22.79 \\
\hline 82 & 4.788 & 27.961 \\
\hline 83 & 27.51 & 36.612 \\
\hline 84 & 26.306 & 15.776 \\
\hline 85 & 4.545 & 25.257 \\
\hline 86 & 23.545 & 35.892 \\
\hline 87 & 10.746 & 37.712 \\
\hline 88 & 7.405 & 6.656 \\
\hline 89 & 12.021 & 6.684 \\
\hline 90 & 29.14 & 27.123 \\
\hline 91 & 23.367 & 29.856 \\
\hline 92 & 14.87 & 16.629 \\
\hline 93 & 11.961 & 17.137 \\
\hline 94 & 16.126 & 18.34 \\
\hline 95 & 22.426 & 16.075 \\
\hline 96 & 19.729 & 10.752 \\
\hline 97 & 19.707 & 7.63 \\
\hline 98 & 23.824 & 21.511 \\
\hline 99 & 8.257 & 27.817 \\
\hline 100 & 9.179 & 24.151 \\
\hline
\end{tabular}


TABLE 6: Results for test problem \#5.

\begin{tabular}{lc}
\hline Program & Cost function value \\
\hline VIP-PLANOPT (2006) [8] & 1084451 \\
GOT & 1062080 \\
\hline
\end{tabular}

The value of overlap area $A_{i j}$ is a nonnegative number. $A_{i j}$ will be zero only if there is no overlapping between facilities $i$ and $j$. The objective is to minimize material handling costs. So, the problem can be stated as follows:

$$
\text { minimizing cost }=\sum_{i=1}^{N-1} \sum_{j=i+1}^{N} f_{i j} d_{i j}
$$$$
\text { subject to } A_{i j}=0 \quad \forall i, j, i<j \text {. }
$$

The constraint ensures that facilities do not overlap. A similar formulation also can be found in [7].

\section{Obtaining an Initial Solution}

The initial solution is obtained by graph theoretic facility layout approaches (GTFLP) and graph drawing algorithms. The following subsection describes obtaining an MPG. Section 3.2 describes the drawing of the MPG on the plane.

3.1. Generating a Maximal Planar Graph. In GTFLP, facilities are represented by vertices, and flow (adjacency desirability) between them is represented by weighted edges. Created graph is called adjacency graph. Graph theory is particularly useful for the facility layout problems, because graphs easily enable us to capture the adjacency information and model the problem. A review of graph theory applications to the facility layout problem can be found in $[9,10]$. GTFLP consists of two stages. At the first stage, the adjacency graph is converted to a maximal planar graph (MPG). In the second stage, a block layout is constructed from the MPG. The second stage is not our concern here. For more details, we refer to [11-15]. Figure 1 shows an MPG and its correspondent block layout.

Many heuristic and metaheuristic methods for obtaining an MPG have been suggested [16-21]. In this paper, we use from the greedy heuristic [16]. It is conceptually simple and creates high weighted MPGs [16]. This heuristic has a simple instruction: the edges are sorted in nonincreasing order of weight. Each edge is tested in turn and accepted as part of the MPG unless it makes the graph nonplanar. So, the heuristic needs planarity testing. Boyer and Myrvold [22] developed a simplified $O(n)$ planarity testing algorithm. We use this algorithm for planarity testing. In the worst case, $O\left(n^{2}\right)$ edges are considered, and for each edge, the Boyer and Myrvold test is called. Hence, the approach results in a complexity of $O\left(n^{3}\right)$.

3.2. Drawing Maximal Planar Graph on the Plane. Graph drawing, as a branch of graph theory, applies topology and geometry to derive two-dimensional representations of graphs. A graph drawing algorithm reads as input a combinatorial description of a graph $G$ and produces as output
TABLE 7: For test problem \#5, the coordinates of facilities obtained by GOT are given below. The layout cost is 1062080 .

\begin{tabular}{|c|c|c|}
\hline Facility & $X$ & $Y$ \\
\hline 1 & 28.617 & 19.513 \\
\hline 2 & 25.371 & 22.082 \\
\hline 3 & 27.683 & 32.088 \\
\hline 4 & 6.488 & 13.531 \\
\hline 5 & 17.53 & 34.357 \\
\hline 6 & 10.131 & 16.929 \\
\hline 7 & 29.906 & 25.226 \\
\hline 8 & 28.243 & 36.473 \\
\hline 9 & 14.372 & 31.298 \\
\hline 10 & 37.502 & 22.299 \\
\hline 11 & 32.586 & 4.846 \\
\hline 12 & 19.349 & 13.249 \\
\hline 13 & 3.314 & 23.588 \\
\hline 14 & 46.96 & 19.354 \\
\hline 15 & 21.953 & 8.539 \\
\hline 16 & 34.13 & 25.537 \\
\hline 17 & 33.636 & 32.8 \\
\hline 18 & 37.21 & 17.564 \\
\hline 19 & 12.603 & 23.713 \\
\hline 20 & 21.763 & 21.428 \\
\hline 21 & 16.261 & 37.283 \\
\hline 22 & 27.453 & 25.185 \\
\hline 23 & 25.213 & 15.36 \\
\hline 24 & 27.111 & 41.844 \\
\hline 25 & 21.208 & 24.959 \\
\hline 26 & 33.287 & 38.471 \\
\hline 27 & 25.882 & 19.125 \\
\hline 28 & 43.87 & 14.147 \\
\hline 29 & 27.342 & 6.265 \\
\hline 30 & 2.831 & 26.3 \\
\hline 31 & 18.328 & 31.432 \\
\hline 32 & 38.33 & 7.761 \\
\hline 33 & 22.131 & 27.038 \\
\hline 34 & 7.166 & 26.77 \\
\hline 35 & 18.909 & 46.302 \\
\hline 36 & 15.123 & 34.383 \\
\hline 37 & 28.974 & 22.602 \\
\hline 38 & 18.728 & 4.656 \\
\hline 39 & 33.526 & 8.657 \\
\hline 40 & 12.176 & 32.318 \\
\hline 41 & 16.539 & 39.732 \\
\hline 42 & 28.383 & 39.485 \\
\hline 43 & 15.09 & 23.899 \\
\hline 44 & 32.571 & 12.559 \\
\hline 45 & 41.221 & 40.78 \\
\hline 46 & 24.335 & 27.843 \\
\hline 47 & 2.516 & 30.62 \\
\hline 48 & 37.452 & 25.455 \\
\hline
\end{tabular}


TABLE 7: Continued.

\begin{tabular}{|c|c|c|}
\hline Facility & $X$ & $Y$ \\
\hline 49 & 40.189 & 36.294 \\
\hline 50 & 12.672 & 36.825 \\
\hline 51 & 34.282 & 27.349 \\
\hline 52 & 19.126 & 15.005 \\
\hline 53 & 36.395 & 41.322 \\
\hline 54 & 31.473 & 40.034 \\
\hline 55 & 30.991 & 34.983 \\
\hline 56 & 38.702 & 27.03 \\
\hline 57 & 28.176 & 12.73 \\
\hline 58 & 29.219 & 45.218 \\
\hline 59 & 25.113 & 10.766 \\
\hline 60 & 11.021 & 27.029 \\
\hline 61 & 22.158 & 14.239 \\
\hline 62 & 22.543 & 17.582 \\
\hline 63 & 12.348 & 21.322 \\
\hline 64 & 13.684 & 43.96 \\
\hline 65 & 8.569 & 36.149 \\
\hline 66 & 8.828 & 33.239 \\
\hline 67 & 10.933 & 12.373 \\
\hline 68 & 24.203 & 26.047 \\
\hline 69 & 12.647 & 7.071 \\
\hline 70 & 29.789 & 27.362 \\
\hline 71 & 6.492 & 38.642 \\
\hline 72 & 18.394 & 17.993 \\
\hline 73 & 4.815 & 17.754 \\
\hline 74 & 24.465 & 45.646 \\
\hline 75 & 24.704 & 24.448 \\
\hline 76 & 48.912 & 31.164 \\
\hline 77 & 19.063 & 11.088 \\
\hline 78 & 27.843 & 16.379 \\
\hline 79 & 35.271 & 29.217 \\
\hline 80 & 21.659 & 35.429 \\
\hline 81 & 15.303 & 28.087 \\
\hline 82 & 32.599 & 31.07 \\
\hline 83 & 3.311 & 20.836 \\
\hline 84 & 44.02 & 33.76 \\
\hline 85 & 43.843 & 9.862 \\
\hline 86 & 16.694 & 23.899 \\
\hline 87 & 20.628 & 17.23 \\
\hline 88 & 19.088 & 35.563 \\
\hline 89 & 48.998 & 25.308 \\
\hline 90 & 33.559 & 46.176 \\
\hline 91 & 31.162 & 30.86 \\
\hline 92 & 24.82 & 36.607 \\
\hline 93 & 44.673 & 24.381 \\
\hline 94 & 18.385 & 27.694 \\
\hline 95 & 30.086 & 10.858 \\
\hline 96 & 33.289 & 15.156 \\
\hline 97 & 30.399 & 17.188 \\
\hline 98 & 15.52 & 19.071 \\
\hline 99 & 36.518 & 32.041 \\
\hline 100 & 19.439 & 40.84 \\
\hline 101 & 22.932 & 4.334 \\
\hline
\end{tabular}

TABle 7: Continued.

\begin{tabular}{lcc}
\hline Facility & $X$ & $Y$ \\
\hline 102 & 27.279 & 28.155 \\
103 & 31.849 & 19.046 \\
104 & 11.48 & 19.243 \\
105 & 7.31 & 21.826 \\
106 & 38.088 & 12.744 \\
107 & 22.892 & 30.522 \\
108 & 14.917 & 13.713 \\
109 & 6.995 & 30.065 \\
110 & 40.466 & 30.376 \\
111 & 41.964 & 19.47 \\
112 & 30.3 & 14.954 \\
113 & 19.953 & 27.768 \\
114 & 27.316 & 2.155 \\
115 & 35.983 & 36.293 \\
116 & 44.374 & 28.853 \\
117 & 22.621 & 41.026 \\
118 & 31.99 & 20.775 \\
119 & 11.309 & 39.88 \\
120 & 41.379 & 24.172 \\
121 & 31.589 & 26.996 \\
122 & 18.031 & 8.178 \\
123 & 18.549 & 22.682 \\
124 & 31.64 & 23.551 \\
125 & 33.452 & 23.294 \\
\hline & &
\end{tabular}

a drawing of G. A graph has infinitely many different drawings. For a review of various graphs drawing algorithms, refer to [23]. We use algorithm of Chrobak and Payne [24] to form a straight line drawing, of the MPG. In such a drawing, each edge is drawn using a straight line segment. The algorithm draws vertices in an MPG to integer coordinates in a $(2 \mathrm{~N}-$ $4) \times(N-2)$ grid. Figure 2 shows an example of straight line drawing.

For acquiring an initial solution, each vertex is replaced by its correspondent facility. In a feasible solution, facilities have no overlaps. For this reason, the coordinates of facilities can be multiplied by maximum dimensions of all facilities (width and length). This operation increases distance between facilities and makes the solution feasible. For the case of circular facilities, the diameter of circle can be considered as maximum dimensions.

\section{Improving Initial Solution and Comparing}

To evaluate the performance, the initial solution is improved by an analytical method by Mir and Imam [7]. In this method, the convergence is controlled by carrying out the optimization using concept of "magnified envelop blocks." The dimensions of the blocks are determined by multiplying the dimensions of the facilities with a "magnification factor." The optimization is then carried out for these envelop blocks rather than the actual facilities. The analytical method searches the optimum placements of each envelop block in 
TABLE 8: The value of cost function in GOT initial solution and the best value of random placements.

\begin{tabular}{|c|c|c|}
\hline$n$ & GOT initial solution & The best random initial solution \\
\hline 10 & 1945 & 2437.3 \\
\hline 11 & 2122 & 2758.5 \\
\hline 12 & 2368 & 3531.7 \\
\hline 13 & 4919 & 5764 \\
\hline 14 & 5514 & 7024 \\
\hline 15 & 5538 & 7449 \\
\hline 16 & 8116 & 10287.7 \\
\hline 17 & 8468 & 12207.5 \\
\hline 18 & 10648 & 15304 \\
\hline 19 & 13943 & 19119.3 \\
\hline 20 & 14265 & 20094 \\
\hline 21 & 17199 & 22491.2 \\
\hline 22 & 16610 & 26370 \\
\hline 23 & 22128 & 30749.2 \\
\hline 24 & 25533 & 35508 \\
\hline 25 & 27585 & 37968.4 \\
\hline 26 & 29929 & 43803.6 \\
\hline 27 & 36255 & 51850 \\
\hline 28 & 41483 & 57050.5 \\
\hline 29 & 47543 & 65109.2 \\
\hline 30 & 57830 & 73705.8 \\
\hline 31 & 61408 & 82058.4 \\
\hline 32 & 63687 & 86436 \\
\hline 33 & 59970 & 94066.4 \\
\hline 34 & 82721 & 110195.2 \\
\hline 35 & 76220 & 104940 \\
\hline 36 & 92426 & 124186.1 \\
\hline 37 & 95386 & 125468 \\
\hline 38 & 87532 & 150060 \\
\hline 39 & 93708 & 146793.8 \\
\hline 40 & 118266 & 180538 \\
\hline 41 & 141363 & 183911 \\
\hline 42 & 104263 & 195458.7 \\
\hline 43 & 152188 & 204207.3 \\
\hline 44 & 163529 & 239253 \\
\hline 45 & 166360 & 237051.8 \\
\hline 46 & 167002 & 251049.3 \\
\hline 47 & 189027 & 290085 \\
\hline 48 & 226097 & 305900 \\
\hline 49 & 234811 & 324500.3 \\
\hline 50 & 238855 & 337198.9 \\
\hline 51 & 264842 & 358480.5 \\
\hline 52 & 251009 & 358666.7 \\
\hline 53 & 233805 & 370262.4 \\
\hline 54 & 298806 & 404104.4 \\
\hline 55 & 284543 & 431091.9 \\
\hline 56 & 344755 & 481656.9 \\
\hline 57 & 371962 & 493986.4 \\
\hline
\end{tabular}

TABLE 8: Continued.

\begin{tabular}{|c|c|c|}
\hline$n$ & GOT initial solution & The best random initial solution \\
\hline 58 & 344044 & 510976 \\
\hline 59 & 375321 & 503358.9 \\
\hline 60 & 373118 & 538554.9 \\
\hline 61 & 370817 & 573555.9 \\
\hline 62 & 457275 & 628602.9 \\
\hline 63 & 544350 & 688184.6 \\
\hline 64 & 530408 & 708518.3 \\
\hline 65 & 502322 & 690275.2 \\
\hline 66 & 526230 & 741481.1 \\
\hline 67 & 556568 & 773325.9 \\
\hline 68 & 639735 & 867242.4 \\
\hline 69 & 578533 & 860229.8 \\
\hline 70 & 643592 & 914437.3 \\
\hline 71 & 589557 & 893978.8 \\
\hline 72 & 670866 & 950592.5 \\
\hline 73 & 736669 & 1058290.5 \\
\hline 74 & 660395 & 1039023 \\
\hline 75 & 749795 & 1115704.6 \\
\hline 76 & 835418 & 1180300 \\
\hline 77 & 727648 & 1157821.9 \\
\hline 78 & 852689 & 1147676 \\
\hline 79 & 971135 & 1273329.8 \\
\hline 80 & 920522 & 1294614.8 \\
\hline 81 & 882645 & 1420567 \\
\hline 82 & 1084711 & 1500037.8 \\
\hline 83 & 1072241 & 1436365.1 \\
\hline 84 & 1072132 & 1606669.3 \\
\hline 85 & 1154018 & 1552139.2 \\
\hline 86 & 1150925 & 1756302.3 \\
\hline 87 & 1164220 & 1608156.3 \\
\hline 88 & 1230008 & 1726587.4 \\
\hline 89 & 1379479 & 1913688.3 \\
\hline 90 & 1351210 & 1970899.3 \\
\hline 91 & 1275975 & 1895126.4 \\
\hline 92 & 1360771 & 2114230 \\
\hline 93 & 1520228 & 1969614.1 \\
\hline 94 & 1542740 & 2125200 \\
\hline 95 & 1581145 & 2230222.7 \\
\hline 96 & 1640792 & 2305569.3 \\
\hline 97 & 1486796 & 2362639.4 \\
\hline 98 & 1645889 & 2365109.3 \\
\hline 99 & 1607866 & 2490733.7 \\
\hline 100 & 2073979 & 2635666.4 \\
\hline
\end{tabular}

the direction of steepest descent which is opposite to the gradient direction. The sizes of the envelop blocks are then reduced, and the optimization process is repeated for the second phase. The number of optimization phases is equal to the magnification factor number for the envelop blocks. In the last optimization phase, the dimensions of the envelop blocks become equal to the actual facilities. For more detail, we refer to Mir and Imam [7]. 
TABLE 9: Summary of the results.

\begin{tabular}{lcccc}
\hline Problem & Number of facilities & Best result by other methods & GOT & Cost reduction \\
\hline$\# 1$ & 8 & 692.5 & 752.7 & -60.2 \\
$\# 2$ & 20 & 1157 & 1302 & -145 \\
$\# 3$ & 50 & 78224.7 & 76882.3 & 1342.4 \\
$\# 4$ & 100 & 538193.1 & 527094.1 & 11099 \\
$\# 5$ & 125 & 1084451 & 1062080 & 22371 \\
\hline
\end{tabular}

So, the proposed approach for solving a facility layout problem can be summarized as follows.

Step 1: encapsulating facilities in envelop blocks (multiplying the dimensions of facilities by a magnification factor).

Step 2: obtaining an MPG.

Step 3: drawing the MPG on the plane and obtaining an initial solution.

Step 4: improving initial solution by analytical method.

Figure 3 shows summary of these steps.

The proposed approach was coded using the VB.NET programming language in a program named GOT (Graph optimization technique). Five test problems were run. For all test problems, results were obtained on a PC with Intel T5470 processor. The results were compared with the previously published papers and commercial software VIP-PLANOPT 2006. VIP-PLANOPT is a useful layout software package that can generate near-optimal layout [25]. For more details about VIP-PLANOPT, see Engineering Optimization Software [8]. VIP-PLANOPT results were obtained from the software user's manual. The results are presented in the following sections.

4.1. Test Problem \#1. This problem of 8 facilities was introduced by Imam and Mir [5]. Figure 4 shows the steps for accruing the initial solution. Figure 4(a) shows the flow matrix and dimension of facilities. All dimensions and cost matrix elements are integer-valued numbers ranging between 1 and 6 . There are several pairs of facilities with no flow between them. Distance norm is squared Euclidean. The greedy heuristic generates the edges lists of MPG as shown in Figure 4(b).

The straight line drawing algorithm gives the coordinates of vertices. The drawing is shown in Figure 4(c). Then, each vertex is replaced by its correspondent facility. The coordinates are multiplied by maximum dimensions of facilities (width and length), and finally, the initial layout design is shown in Figure 4(d).

The solution is improved by the analytical technique. Figure 5 shows the final layout. The cost function value for this layout is 752.7, and the running time is 0.4 second. Table 1 shows the results obtained by the other approaches. The best solution for this problem is obtained by VIP-PLANOPT 2006.
4.2. Test Problem \#2. This problem of 20 unequal area facilities was introduced by Imam and Mir [6]. The data consist of only integer values. The dimensions of the facilities are between 1 and 3 . The elements of the cost matrix are integers between 0 and 5 . The distance norm is rectilinear. The final layout obtained by GOT is shown in Figure 6. The layout cost is 1302, and the running time is 0.6 second. Table 2 compares the results obtained by GOT with the results available in the literature. VIP-PLANOPT 2006 has the lowest value of the cost function.

4.3. Test Problem \#3. This is a problem of 50 facilities randomly generated by VIP-PLANOPT 2006. The dimensions of the facilities are decimal numbers between 1 to 6 . The elements of the cost matrix are all integers between 1 and 10. The distance norm is Euclidean. The results are shown in Table 3. The best published result has a cost of 78224.7, whereas GOT produces a final layout with a cost of 76882.3 only in 15.1 seconds. Figure 7 shows the final layout.

4.4. Test Problem \#4. This is a randomly generated large size problem of 100 facilities. The dimensions of the facilities are decimal numbers between 1 and 6 . The cost matrix elements are integers between 1 and 10 . The distance norm is rectilinear. The results are shown in Table 4. GOT obtained the cost function value of 527094.1 in 74.3 seconds. This value is about $2 \%$ below the cost function value of VIP-PLANOPT 2006. The coordinates of the facilities for the layout obtained by GOT are given in Table 5.

4.5. Test Problem \#5. This is a large size problem of 125 facilities randomly generated by VIP-PLANOPT 2006. The dimensions of facilities are real numbers between 1 and 6 , and elements of the cost matrix are integers between 1 and 10 . The distance norm is rectilinear. The results are shown in Table 6. GOT obtained the cost function value of 1062080 in 129.6 seconds. This value is about $2 \%$ below the cost function value of VIP-PLANOPT 2006. The coordinates of the facilities for the layout obtained by GOT are given in Table 7 .

\section{Comparing GOT Initial Solution with Random Initial Solution}

To compare the proposed initial solution (GOT initial solution) with random initial solution, a set of test problems $(n=$ $10,11,12, \ldots, 100)$ were generated. The facilities dimensions were $1 \times 1$, and flow matrices were randomly generated between 0 and 10 . For acquiring a random initial solution, 


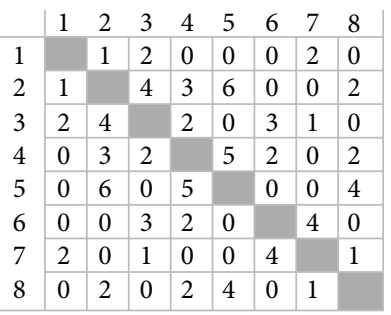

Flow matrix

\begin{tabular}{|c|c|c|}
\hline & Length & Width \\
\hline & 2 & 3 \\
\hline & 4 & 5 \\
\hline & 2 & 2 \\
\hline & 3 & 3 \\
\hline & 2 & 4 \\
\hline & 4 & 4 \\
\hline & 4 & 4 \\
\hline & 3 & 4 \\
\hline
\end{tabular}

Dimensions of the facilities

(a) The raw facilities layout data
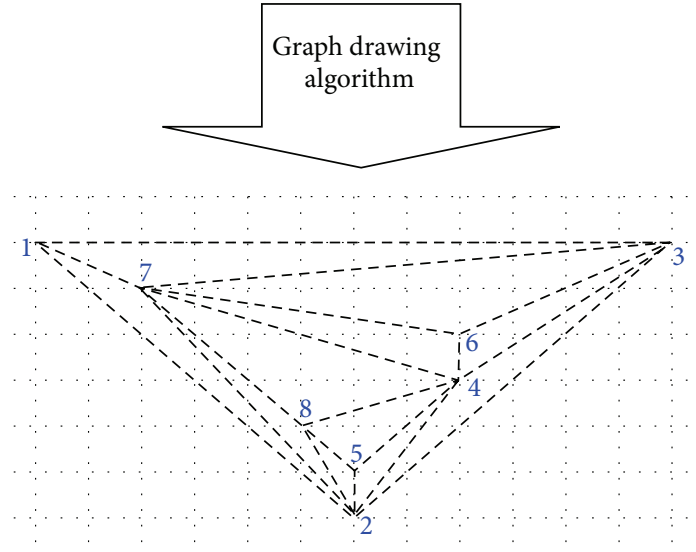

(c) Straight line drawing of the MPG

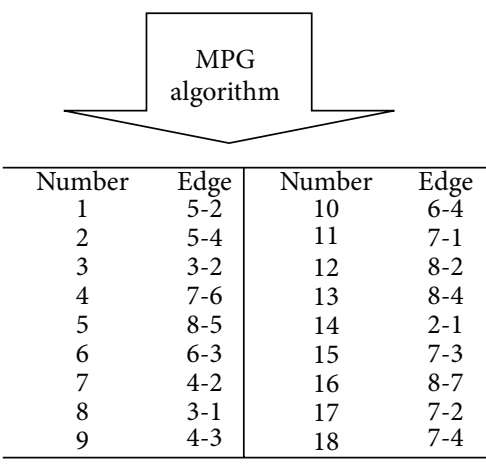

(b) MPG

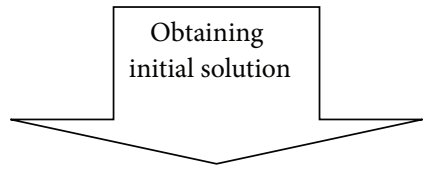

7

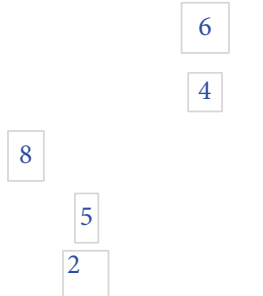

(d) Initial solution

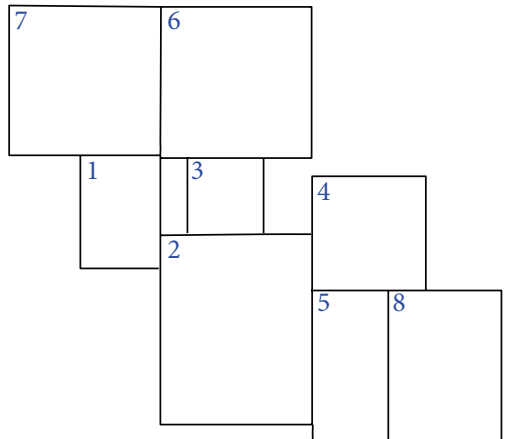

FIGURE 5: Final layout for test problem \#1.

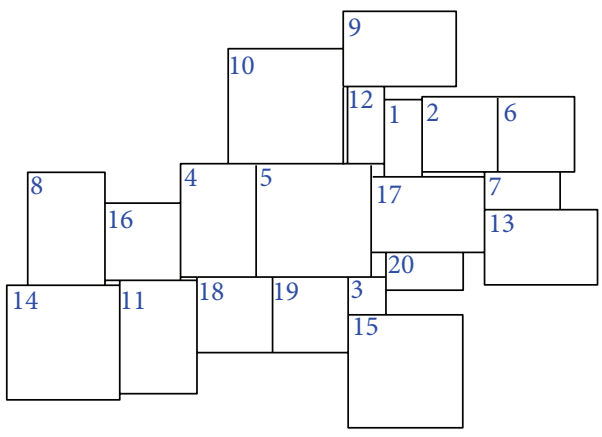

FIGURE 6: Final layout for test problem \#2. facilities were randomly placed in a $(2 n-4) \times(n-2)$ integer grid. For each test problem, 20 random placements were found. Table 8 shows the value of cost function in GOT initial solution and the best value found by random placements. Figures 8 and 9 shows these results graphically. The results demonstrated significant improvement in cost function.

\section{Summary and Conclusion}

An initial solution has been presented for the layout design of facilities on a continuous plane. The technique consists of two stages. In the first stage, a maximal planar graph (MPG) is obtained. In the second stage, the vertices of MPG are drawn on the plane by graph drawing algorithms. Then, vertices are replaced by facilities. Hence, an initial solution is obtained. To evaluate the performance, this initial solution has been applied in rectangular facility layout problem and improved by an analytical method by Mir and Imam [7].

The approach has been tested on five instances from the literature. Table 6 shows the Summary of the results, and Figure 8 shows the cost reduction by the technique. For the large size problems involving 50, 100, and 125 facilities, 


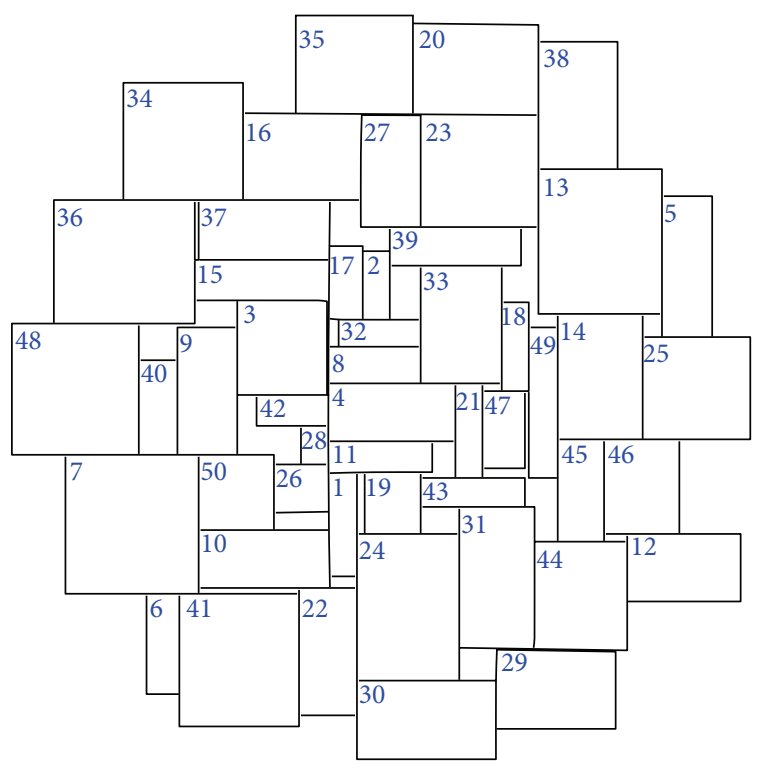

Figure 7: Final layout for test problem \#3.

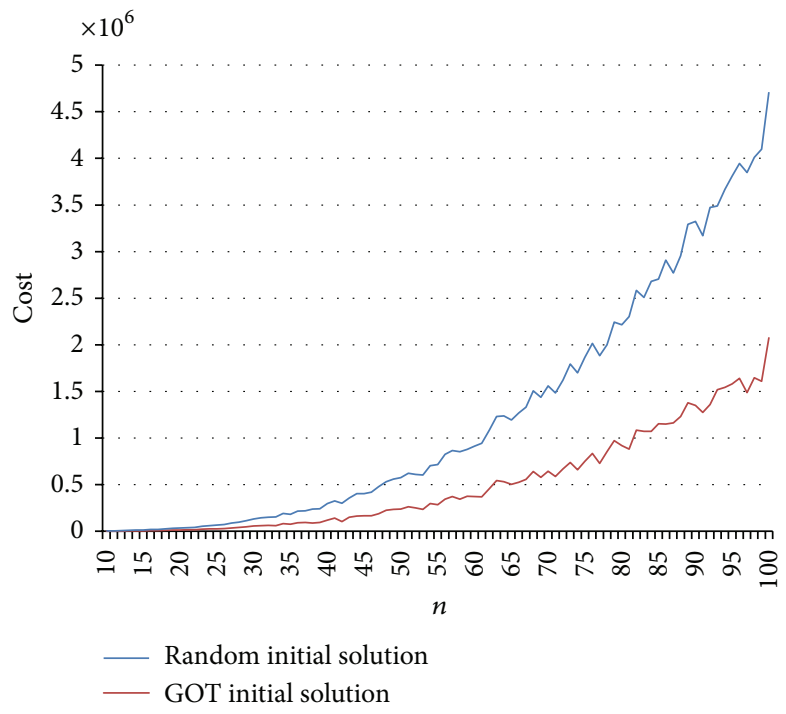

FIgURE 8: Comparisons of cost function value in GOT initial solution with the best value of random placements.

the layout costs values are better than those obtained by the previously published techniques. As shown in Table 9, the results demonstrate effectiveness of the technique, especially for large size problems.

This paper introduced a simple technique for obtaining a good initial solution. The technique, with some modification, can be applied in facility layout approaches that use a randomly generated initial solution. In future researches, it would be interesting to analyze the influence of MPG and graph drawing algorithm on the solution. The results can be further improved by using a metaheuristic such as GRASP [21] and Tabu search [20] for generating a high weighted MPG.

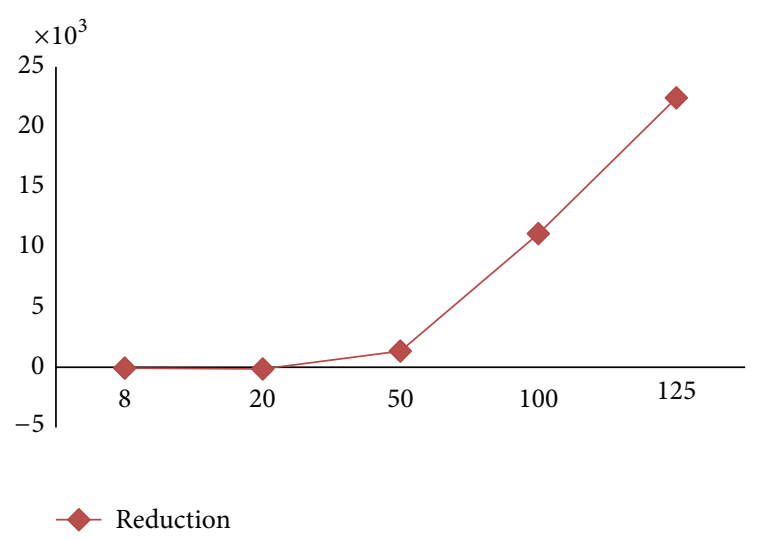

Figure 9: Cost reduction by using GOT.

\section{Conflict of Interests}

The authors declare that they have no conflict of interests.

\section{References}

[1] S. P. Singh and R. R. K. Sharma, "A review of different approaches to the facility layout problems," International Journal of Advanced Manufacturing Technology, vol. 30, no. 5-6, pp. 425-433, 2006.

[2] M. F. Anjos and A. Vannelli, "An attractor-repeller approach to floorplanning," Mathematical Methods of Operations Research, vol. 56, no. 1, pp. 3-27, 2002.

[3] I. Castillo and T. Sim, "A spring-embedding approach for the facility layout problem," Journal of the Operational Research Society, vol. 55, no. 1, pp. 73-81, 2004.

[4] Z. Drezner, "DISCON: a new method for the layout problem," Operations Research, vol. 28, no. 6, pp. 1375-1384, 1980.

[5] M. H. Imam and M. Mir, "Nonlinear programming approach to automated topology optimization," Computer-Aided Design, vol. 21, no. 2, pp. 107-115, 1989.

[6] M. H. Imam and M. Mir, "Automated layout of facilities of unequal areas," Computers and Industrial Engineering, vol. 24, no. 3, pp. 355-366, 1993.

[7] M. Mir and M. H. Imam, "Hybrid optimization approach for layout design of unequal-area facilities," Computers and Industrial Engineering, vol. 39, no. 1-2, pp. 49-63, 2001.

[8] Engineering Optimization Software, VIP-PLANOPT, 2006, 2010, http://www.planopt.com/.

[9] L. Foulds, Graph Theory Applications, Springer, New York, NY, USA, 1992.

[10] M. M. D. Hassan and G. L. Hogg, "A review of graph theory application to the facilities layout problem," Omega, vol. 15, no. 4, pp. 291-300, 1987.

[11] M. M. D. Hassan and G. L. Hogg, "On converting a dual graph into a block layout," International Journal of Production Research, vol. 27, no. 7, pp. 1149-1160, 1989.

[12] K. H. Watson and J. W. Giffin, "The vertex splitting algorithm for facilities layout," International Journal of Production Research, vol. 35, no. 9, pp. 2477-2492, 1997.

[13] S. A. Irvine and I. Rinsma-Melchert, "A new approach to the block layout problem," International Journal of Production Research, vol. 35, no. 8, pp. 2359-2376, 1997. 
[14] P. S. Welgama, P. R. Gibson, and L. A. R. Al-Hakim, "Facilities layout: a knowledge-based approach for converting a dual graph into a block layout," International Journal of Production Economics, vol. 33, no. 1-3, pp. 17-30, 1994.

[15] M. A. Jokar and A. S. Sangchooli, "Constructing a block layout by face area," The International Journal of Advanced Manufacturing Technology, vol. 54, no. 5-8, pp. 801-809, 2011.

[16] L. R. Foulds, P. B. Gibbons, and J. W. Giffin, "Facilities layout adjacency determination: an experimental comparison of three graph theoretic heuristics," Operations Research, vol. 33, no. 5, pp. 1091-1106, 1985.

[17] E. G. John and J. Hammond, "Maximally weighted graph theoretic facilities design planning," International Journal of Production Research, vol. 38, no. 16, pp. 3845-3859, 2000.

[18] S. G. Boswell, "TESSA-a new greedy heuristic for facilities layout planning," International Journal of Production Research, vol. 30, no. 8, pp. 1957-1968, 1992.

[19] L. R. Foulds and D. F. Robinson, "Graph theoretic heuristics for the plant layout problem," International Journal of Production Research, vol. 16, no. 1, pp. 27-37, 1978.

[20] I. H. Osman, "A tabu search procedure based on a random Roulette diversification for the weighted maximal planar graph problem," Computers and Operations Research, vol. 33, no. 9, pp. 2526-2546, 2006.

[21] I. H. Osman, B. Al-Ayoubi, and M. Barake, "A greedy random adaptive search procedure for the weighted maximal planar graph problem," Computers and Industrial Engineering, vol. 45, no. 4, pp. 635-651, 2003.

[22] J. M. Boyer and W. J. Myrvold, "On the cutting edge: simplified $\mathrm{O}(\mathrm{n})$ planarity by edge addition," Journal of Graph Algorithms and Applications, vol. 8, no. 3, pp. 241-273, 2004.

[23] G. Di Battista, P. Eades, R. Tamassia, and I. Tollis, Graph Drawing: Algorithms for the Visualization of Graphs, Prentice Hall PTR, Upper Saddle River, NJ, USA, 1998.

[24] M. Chrobak and T. H. Payne, "A linear-time algorithm for drawing a planar graph on a grid," Information Processing Letters, vol. 54, no. 4, pp. 241-246, 1995.

[25] S. Heragu, Facilities Design, Iuniverse Inc., 2006. 


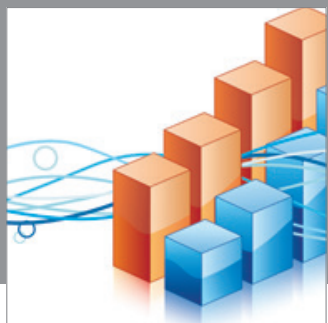

Advances in

Operations Research

mansans

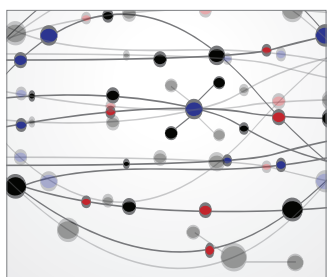

The Scientific World Journal
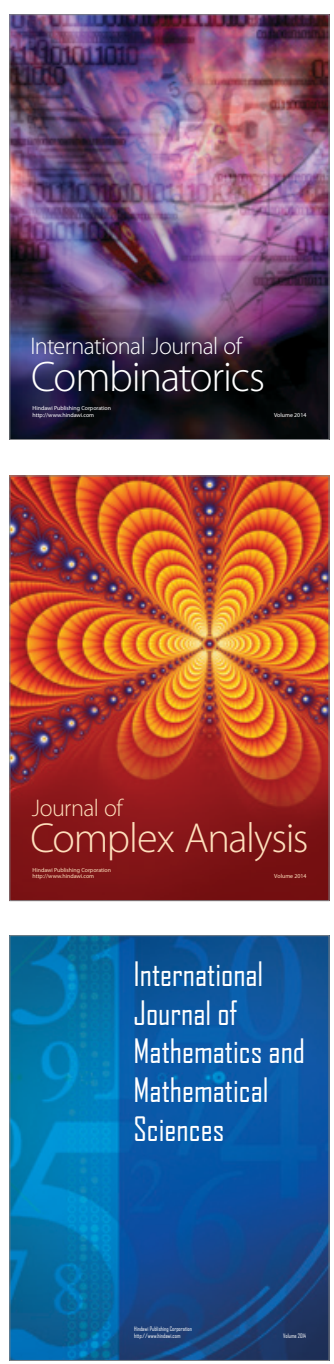
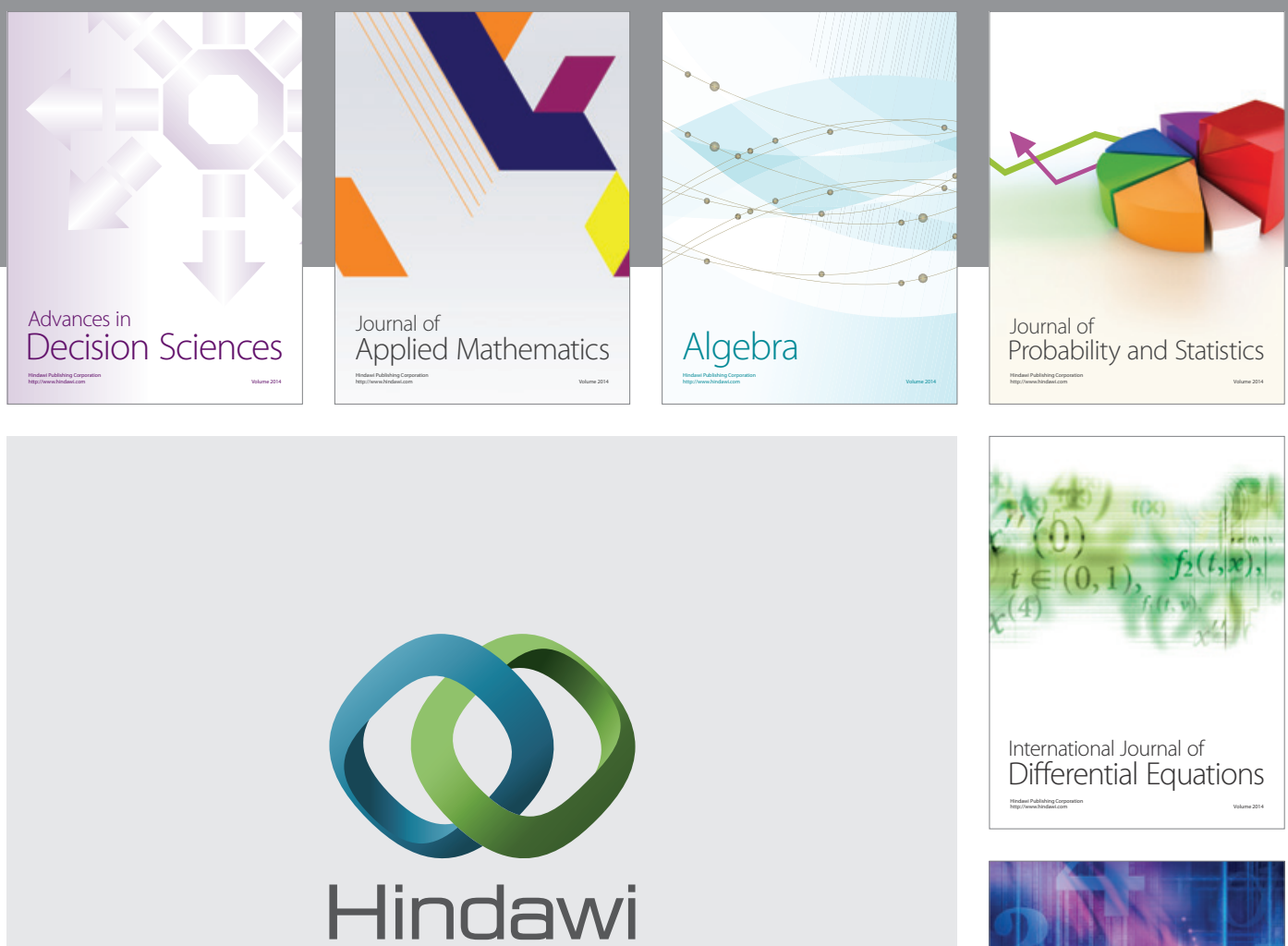

Submit your manuscripts at http://www.hindawi.com
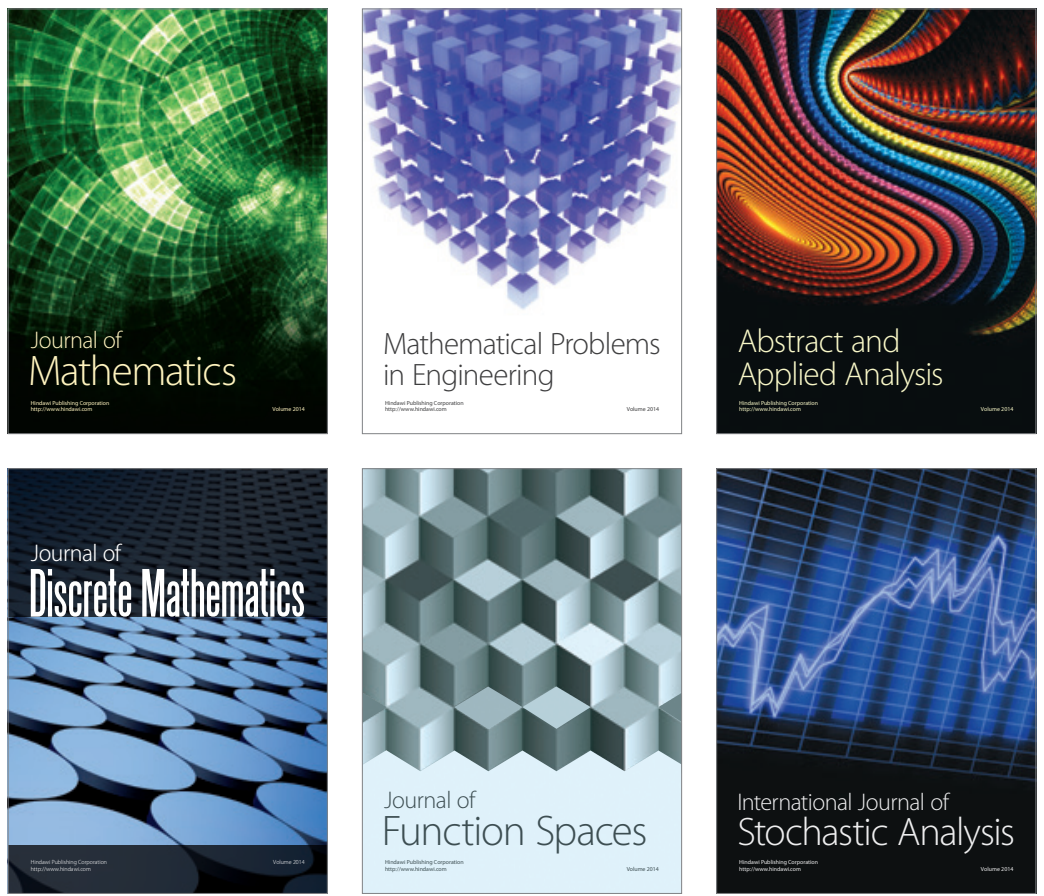

Journal of

Function Spaces

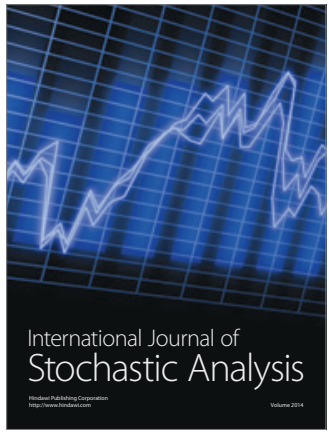

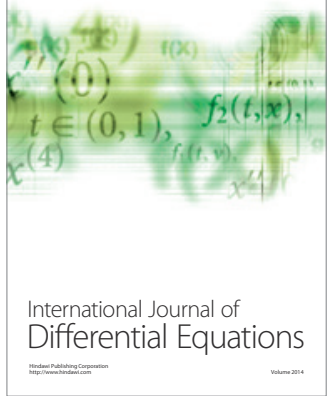
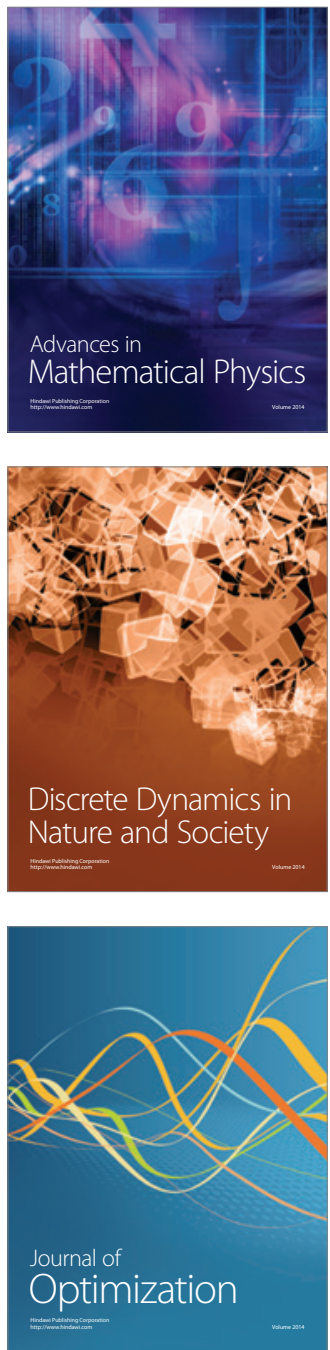